DOSSIER

\title{
Sehgal no invita a la lógica. Performance, experiencia y economía inmaterial en Tino Sehgal
}

\author{
The Illogic of Sehgal. Performance, Experience \\ and Immaterial Economy in Tino Sehgal's Work
}

\author{
Juan Albarrán Diego \\ Universidad Autónoma de Madrid
}

Recibido: diciembre 2016

Aprobado: enero 2017

\begin{abstract}
Resumen
Tino Sehgal es uno de los artistas más reconocidos y aclamados de los últimos años. Su obra se concreta en situaciones (performances) que intérpretes contratados desarrollan en museos, bienales y galerías de todo el mundo. Esas situaciones nunca producen restos materiales (fotografías, objetos, partituras, contratos, etc.) que puedan ser exhibidos, publicados o coleccionados. Este artículo explora algunas de las contradicciones derivadas de la concepción del trabajo artístico en Sehgal, de su posición en el sistema internacional del arte, así como de las estrategias que ha desarrollado para tratar de escapar a las inercias institucionales y mercantiles en él dominantes.
\end{abstract}

Palabras clave: comisariado, experiencia, participación, performance.

\begin{abstract}
Tino Sehgal is one of the most acclaimed artists of recent years. His work consists of situations interpreted by hired performers. Those situations, designed for museums, biennials and galleries around the world, never produce material traces: photographs, objects, scores or contracts of his pieces could not be displayed, published or collected. This paper explores some of the contradictions related to Sehgal's conceptualization of artistic labour, its position in the international art system, and the strategies that he has developed in order to avoid the hegemonic institutional and commercial inertias.
\end{abstract}

Key Words: curating, experience, participation, performance. 


\section{Introducción: cuando el arte pasa como la vida}

"Luego empezó a interesarme ya más Sehgal, sobre todo cuando vi que su lema principal podría ser éste: "Cuando el arte pasa como la vida». Sehgal proponía que sólo participando en su performance uno pudiera decir que había visto la obra. Bien mirado, eso estaba muy bien. Cuando el arte pasa como la vida. Sonaba perfecto" (Enrique Vila-Matas, 2014, p. 54).

Poco importa si el lema de resonancias vanguardistas que Vila-Matas atribuye a Sehgal ha salido o no de la boca del artista. En efecto, uno de los objetivos de su trabajo pasa por retensionar las relaciones entre nuestra cotidianidad y ese conjunto de prácticas, dispositivos y discursos a los que nos referimos como arte. $\mathrm{Y}$ eso es, precisamente, lo que interesa a Vila-Matas, que en varios puntos de la novela - concebida como un relato sobre su estancia en la dOCUMENTA 13 de Kassel (2012) - narra su experiencia al confrontarse con la pieza de Sehgal This Variation: una sala a oscuras en la que el visitante comparte tiempo, espacio y situaciones con performers cuya presencia percibe, pero a los que no puede ver. Esas insólitas situaciones fascinan al escritor, que encuentra en ellas un pretexto para divagar sobre las fuentes de la creatividad y el sentido del arte. Tampoco importa demasiado si el texto de Vila-Matas guarda alguna semejanza con la realidad de su experiencia en la ciudad alemana. No solo porque su obra se asiente en el inestable filo que separa realidad y ficción, sino también porque, en lo relativo a Sehgal, "sólo participando en su performance uno pudiera decir que había visto la obra", lo cual desaconseja tomar en consideración aquello que otros puedan contarnos sobre ella.

Aquí radica una de las claves del trabajo del artista, nacido en Londres y asentado en Berlín. Supuestamente, sus situaciones construidas - como prefiere llamar a sus performances - solo pueden ser experimentadas "en directo". La copresencia de los cuerpos de público y performers contratados por Sehgal constituye la obra. Es decir, sus performances no arrojan restos materiales que permitan acceder a su trabajo, que puedan ser expuestos o coleccionados. Y la obra no es una idea que, tal y como propusiese Weiner, puede o no ser realizada. Por supuesto, la "idea" — su método de trabajo, el modo de desarrollar y comunicar sus proyectos - está en la base de su actual fortuna crítica y, en un nivel discursivo que el artista no puede eludir, forma parte de la pieza. Pero lo decisivo se ventila en el espacio expositivo, cuando el visitante entra en contacto con sus situaciones. Esa experiencia irremplazable es la pieza. Irremplazable porque no podría ser sustituida por la información contenida en un catálogo o por un vídeo o conjunto fotográfico. Sehgal no documenta sus piezas; no comercializa, por tanto, fotografías o vídeos de sus situaciones, como hacen otros muchos artistas; no existen monografías sobre su trabajo y nunca incluye imágenes en los catálogos de las exposiciones colectivas en las que participa. En la guía de la 
referida dOCUMENTA 13, las páginas que, tal y como indica el índice, debían estar reservadas a su obra han desaparecido del volumen (Christov-Bakargiev, 2012, pp. 438-439). Con todo ello, Sehgal apuntala la mitología de la presencia que ha envuelto a las prácticas performativas desde, al menos, los años sesenta y propone modos no convencionales de exposición y circulación del hecho artístico. En este texto, me propongo explorar algunas de las contradicciones derivadas de la concepción del trabajo artístico en Sehgal, de su posición en el sistema internacional del arte, así como de las estrategias que ha desarrollado para tratar de escapar a las inercias institucionales y mercantiles en él dominantes.

\title{
2. En directo, otra vez
}

\begin{abstract}
"Sehgal, me recordó Boston, rechazaba la idea de que el arte tuviera una expresión física, es decir, que fuera un cuadro, escultura, artefacto, instalación, etc., y trataba con igual desdén la idea de una explicación escrita de su obra. Por tanto, tal como ya me había dicho antes, la única forma de poder contar que uno había visto una obra de Sehgal era verla en directo" (E. Vila-Matas, 2014, p. 55).
\end{abstract}

En noviembre de 2015 pude ver "en directo" la exposición —así la presentaba el museo, comisariada por Beatrix Ruf y Martijn van Nieuwenhuyzen - de Tino Sehgal en el Stedelijk Museum de Amsterdam. Tino Sehgal. A Year in the Stedelijk ocupó algunos de los espacios destinados a acoger la presentación de su colección desde el 1 de enero al 31 de diciembre de 2015. El proyecto se concibió como una sucesión de situaciones en doce entregas: cada mes variaba el número de performances que el espectador podía experimentar en las salas del museo. Es decir, cada mes el visitante que se acercase al Stedelijk se encontraba con una retrospectiva distinta y sólo si acudía al museo todos los meses del año podía "participar" la exposición completa. El número de performances desarrolladas en el museo se fue incrementando durante la primera mitad del año y decreció lentamente durante la segunda mitad, por lo que en mi visita sólo pude encontrar tres de las piezas: This is Propaganda (2002), Selling Out (2002) y This is Critique (2008). Trataré de referirme, pues, a las situaciones a las que asistí en el Stedelijk desde los recuerdos de mi experiencia, que empezaron a quedar fijados por escrito en este documento en un hotel cercano al museo pocas horas después de mi visita.

La primera pieza con que me topé aquella mañana fue el estriptis de un joven vigilante de sala (Selling Out) que empezaba a voltear su chaqueta por el aire, sin ningún respeto por las grandes piezas de madera de Carl Andre (Bloody Angel, 1985) que ocupaban la esquina opuesta de la sala. Después de unos segundos de desconcierto, de manera casi inconsciente, encendí la pequeña cámara digital que llevaba entre mis manos. De inmediato, el performer me miró fijamente: "no photos". El joven vigilante siguió desnudándose con su baile erótico hasta quedarse 
en ropa interior. En ese momento, me di cuenta de que detrás de mí, en la sala que acababa de atravesar, había un segundo vigilante. Pensé que debía pertenecer también a la obra. Me acerqué y le pregunté si era parte de la performance. Me dijo que era "This is Critique, Tino Sehgal, 2008", y acto seguido me preguntó qué me había parecido lo que acababa de ver: "Shocking and a bit embarrassing". Le pregunté qué formación tenían quienes trabajaban poniendo en escena las piezas de Sehgal. Mostrando su incomodidad por mi pregunta, respondió con desgana que no lo sabía y que no tenía importancia. Me despedí y seguí la voz que a lo lejos anunciaba la tercera de las situaciones. Una vigilante de sala cantaba frente a un gran monocromo: "This is propaganda, this is propaganda. You know, you know. This is propaganda...". Tras escuchar durante un par de minutos, salí de la sala y continué mi recorrido por la colección del museo. Paré para comer en la cafetería, visité la exposición sobre el grupo Zero y volví a las salas donde tenían lugar los trabajos de Sehgal.

Había pasado poco más de una hora y media y los performers habían rotado. Podía experimentar en directo, otra vez, las situaciones, aunque éstas ya eran definitivamente otras. El estríper era ahora una joven. Entré en la sala cuando empezaba a desnudarse. Frente a ella, una pareja adolescente y una mujer de mediana edad miraban el espectáculo sin pestañear. La chica siguió desnudándose de manera muy sensual, interpelándonos con la mirada, provocadora, sugerente. Tiró al suelo su sujetador y cuando parecía que iba a quitarse las bragas se detuvo para desvelarnos que era una obra de Tino Sehgal. Me di la vuelta para hablar con una vigilante que estaba a unos metros. Le expliqué que era profesor de Historia del Arte, que estaba muy interesado en el trabajo de Sehgal y le pregunté — deformación profesional - si me podía contar algo sobre la preparación de esas situaciones: qué formación tenían los intérpretes, cómo los había elegido el artista, etc. Me dijo que se suponía que no debía hablar de eso, que ella era parte de la pieza "This is Critique". Insistí y me comentó que dependía de cada pieza. Los intérpretes habían sido seleccionados en audiciones. Ella estudiaba Bellas Artes. Sehgal les había dado instrucciones para conducir sus conversaciones con los visitantes. Le dije que me daba la impresión de que sus colegas estríperes eran bailarines. Sin querer decirme mucho, me confirmó que algunos eran estríperes profesionales y otros tenían formación en el campo de la danza. Cumpliendo con su trabajo y como había hecho su compañero antes, me preguntó qué me había parecido la situación.

Así, pues, Tino Sehgal genera situaciones que el visitante nunca esperaría encontrar en un museo: un vigilante, responsable de la seguridad y del silencio de las salas, canta y, además, nos descubre que todo lo que vemos es propaganda; otro se desnuda en un espacio en el que podemos encontrar muchos desnudos pictóricos - casi siempre femeninos-, pero en el que no podríamos imaginar una situación tan excitante que, sin embargo, nunca llega a excitarnos; un tercero nos 
interpela, invitándonos a reflexionar y verbalizar una experiencia sobre la que la mayor parte del público no está acostumbrado a conversar, menos si cabe con la propia pieza; todos ellos incorporan la cartela como un elemento que interrumpe la relación con los objetos artísticos, esa información textual que buscamos junto a las obras de arte para situarlas y ordenar nuestra experiencia y que, al mismo tiempo, altera la neutralidad estética del cubo blanco y afirma el valor artístico de las obras. El artista ha sido muy cuidadoso a la hora de capitalizar ese elemento vivencial como una especie de marca de autor. Otra cosa distinta es pensar que sus situaciones puedan pasar, efectivamente, "como la vida misma". De hecho, pretenden ser —quizás lo consiguen - bastante más inteligentes, divertidas y epatantes que cualquier momento de nuestra por lo general tediosa cotidianeidad.

Pese a que el artista no comercializa fotografías de sus trabajos, no siempre se prohíbe al visitante tomar fotos de sus situaciones - la prohibición de fotografiar el estriptis de Selling Out parece lógica-. Pude hacer una foto de This is Propaganda, aunque esa imagen nada nos dice del timbre de voz de la cantante o de la incomodidad que sentían los visitantes al buscar la fuente de esa melodía a través de salas repletas de objetos de arte. No hubiese podido hacer una foto de la conversación de This is Critique, solo podría tratar de transcribirla. Tal vez este escrito sea el único documento posible de mi recorrido por las salas del Stedelijk. Un relato al que, en un marco académico como el de esta revista, se le presupone - no digo que lo tenga - cierto valor de verdad, distinto, por tanto, del valor literario de la novela de Vila-Matas. Un documento textual, en cualquier caso, deficitario con respecto a la información que creemos obtener de los registros audiovisuales de otras performances; deficitario, también, con respecto a las experiencias que viví "en directo": el sudor frío al verme violentado por la mirada de un joven que se desnuda ante mí, la indescriptible sensación de extrañeza al escuchar una voz que cantaba a lo lejos, el impulso que me llevó a preguntar a la joven que interpretaba This is Critique por la producción de las situaciones como quien intenta desvelar el mecanismo lógico de un truco de magia.

Ahora bien, tal y como ha explicado Philip Auslander en su ya clásico Liveness (1999), las experiencias vividas en directo - in-mediatas, no-mediadas - siempre conllevan ciertas dosis de mediación. La oposición entre lo directo-presente, que Sehgal trata de enfatizar, y lo mediatizado, que rechaza por su carácter material, no es ni mucho menos ontológica, sino, antes bien, cultural. En Sehgal resulta evidente el esfuerzo discursivo y, paradójicamente, material que requiere la construcción de la "presencialidad" —liveness-.

\section{Exhibir y coleccionar}

La fotografía ha sido y sigue siendo uno de los medios más usados para exhibir acciones $y$, sin duda, es la principal fuente de información visual para conocer 
la historia de la performance. El occidental medio se relaciona con el mundo a través de la pantalla de su teléfono móvil y puede seguir haciéndolo si lo desea ante las situaciones de Sehgal. En su caso, el veto a la imagen no afecta a su recepción en la sala, sino a su circulación en el sistema del arte. Al fin y al cabo, si la performance solo existe en directo, poco puede importar que el espectador tome fotos que nada dirán de esa experiencia. No podremos, eso sí, adquirir fotos de Sehgal en una galería, consultarlas en un catálogo o encontrarlas expuestas en la sala de un museo. Si, desde los años sesenta, la retórica documental de la fotografía ha contribuido a reforzar la inmaterialidad de la performance (Albarrán, 2012), si, incluso, le ha dado carta de naturaleza como tal (Auslander, 2006, 2014), la imposibilidad de registrar la obra de Sehgal y el carácter inmaterial y presuntamente irremplazable de la experiencia que nos propone tiene varias consecuencias en los niveles expositivo, mercantil y discursivo.

En 2003, cuando todavía no había cumplido treinta años y apenas llevaba tres trabajando en el ámbito del arte, Sehgal imaginaba cómo podría ser una futura revisión expositiva de su obra, en la que no habría vídeos, fotos, objetos o instrucciones: "Me gustaría hacer una exposición individual en un museo totalmente vacío que, al mismo tiempo, estuviese lleno; vacío y lleno dado que no habría objetos, pero sí piezas sucediendo en cada sala, así que, de algún modo, sería muy convencional"(1) (Obrist, 2003). Doce años después, la retrospectiva del Stedelijk fue igualmente anti-objetual, aunque ligeramente diferente. El museo no estaba vacío. Estaba tan "lleno" de objetos artísticos como de costumbre. Es cierto que sucedían piezas en sus salas, pero no podría decirse que la retrospectiva fuese convencional. Tal y como apunta Isabel Ruf, directora del Stedelijk desde 2014, alojar la obra de Sehgal supuso un reto para el museo, cuyo personal y display expositivo tuvieron que adaptarse durante todo un año para atender las necesidades de la muestra (Cahier, 2015). La exposición no se preparó con antelación, como se supone hacen los museos. El proyecto fue tomando forma durante el año en que Sehgal ocupaba las salas del Stedelijk. A ese respecto, Martijn van Nieuwenhuyzen explica:

"pudimos desarrollarlo sobre la marcha con Tino. Cada mes elegíamos nuevos trabajos con su ayuda, y nuevas combinaciones de trabajos a partir de la experiencia que teníamos con las piezas durante el mes anterior. Pudimos desarrollar la exposición de una manera natural. Fue un work in progress, un verdadero diálogo con el artista. Para este proyecto, encontramos el presupuesto en tan sólo tres meses; gracias a su carácter arriesgado, fue generosamente financiado por dos patrocinadores. La organización fue todo un reto" (2) (Kravchuk, 2016).

En los últimos años, otros artistas han experimentado con nuevos formatos expositivos desarrollando estrategias para eludir las convenciones de las retrospectivas de media carrera. Entre ellos, Bishop ha llamado la atención sobre las exposiciones de Rirkrit Tiravanija (Retrospective. Tomorrow is Another 
Fine Day, 2004-2005), Pierre Huyghe (Celebration Park, 2006), Jonathan Monk (Continous Project Altered Daily, 2005) o el bailarín y coreógrafo Xavier Le Roy (Retrospectiva, 2011) — con el cual Sehgal trabajó en sus años de formacióncomo proyectos que cuestionan la re-presentación de propuestas contextuales, participativas o performativas. Desde la voluntad de revisitar el sentido de las obras sin someterlas a procesos de escenificación o reconstrucción museal, estos artistas han encontrado fórmulas muy diversas para reimaginar sus piezas, para traerlas al presente en forma de nuevos trabajos (2014a, pp. 103-108). El año de Sehgal transita esa misma línea de investigación, en que la exposición no se plantea como un dispositivo estable y acumulativo que preserva obras clausuradas, sino como un medio abierto y cambiante que permite tomar contacto con propuestas que vuelven de manera siempre diferente.

En el Stedelijk, como es habitual, las situaciones de Sehgal no se programaban, no empezaban ni terminaban a una hora determinada, sino que se desarrollaban durante todo el tiempo de apertura del museo. Así, la performance se infiltraba en su colección, la parasitaba para connotarla. El centro holandés, a lo largo de su historia, ha destacado por mantener una relación fluida y cercana con los artistas que programaba, pero también por su capacidad para innovar en la conceptualización de sus exposiciones e integrar las prácticas más rupturistas en su prestigiosa colección. La exposición de Sehgal se sitúa en ese espacio discursivo de una manera consciente. El artista conoce las posibilidades y peculiaridades históricas de la institución y las fortalezas de una colección con la cual está dialogando - la elección de las salas en que se desarrolla cada situación no es ni mucho menos casual- Del mismo modo, también la institución está interesada en patrimonializar la propuesta anti-objetual e iconoclasta de Sehgal, experimentar con nuevos dispositivos expositivos y sumar ese capital simbólico a su colección.

El Stedelijk adquirió en 2005 la pieza Instead of Allowing Some Thing to Rise Up to Your Face Dancing Bruce and Dan and Other Things (2000), que fue elegida para abrir, en el mes de enero, el proyecto de Sehgal. Ese mismo año, la Tate incorporó a sus colecciones This is Propaganda (2002). El MoMA compró Kiss (2003) en 2008 por 70.000 dólares. No hay que perder de vista que el centro amsterdamés había sido uno de los pioneros en adquirir arte conceptual. De hecho, como demostrase Sophie Richard, el Setedelijk Museum fue, desde finales de los años sesenta y a lo largo de los setenta, la institución que más activamente coleccionó arte conceptual con más de 62 piezas $(2009$, p. 215). En 1972, el museo ya había invertido importantes sumas de dinero en trabajos de Huebler, Kosuth, Nauman o Darboven, y, entre ese año y 1978, programó individuales de Dibbets, Gilbert and George, Long, LeWitt, Ryman o Buren —uno de los referentes de Sehgal—. Las prácticas conceptuales plantearon en su momento formas alternativas de producir, comunicar y comercializar arte; formas de una objetualidad poco convencional 
que fueron de inmediato asumidas por instituciones y mercado. Sehgal pretende ir un paso más allá. En su caso, no hay contratos, recibos de compra, partituras $\mathrm{u}$ otros documentos que conformen la pieza, permitan reproducirla o determinen su propiedad. Cuando una institución adquiere uno de sus trabajos, Sehgal en persona da instrucciones y negocia condiciones ante representantes del museo, de su galería y un notario, que actúa como testigo de la transacción. En el acto, el museo no recibe nada material. La venta es también una situación construida que se consuma en la transmisión oral de información durante la interacción de cuerpos cuyas memorias conservarán la pieza (Carpenter, 2014). Sin registros ni instrucciones, Sehgal sabe que el paso del tiempo puede alterar la memoria y, por tanto, la esencia experiencial de sus piezas. ¿Qué sucederá cuando todos los presentes en el acto de transacción fallezcan?, ¿cómo se acomodará la pieza a la temporalidad del museo?, ¿cómo se orquestará el re-enactment de cada situación cuando Sehgal o los miembros de su equipo no puedan supervisar el proceso? Es cierto que abundan las descripciones textuales de sus trabajos, y que estos parecen relativamente simples, fáciles de reconstruir. No obstante, hay que tener en cuenta que Sehgal selecciona minuciosamente a sus intérpretes y que, cabe suponer, el museo deberá ser igualmente cuidadoso tanto en el proceso de selección como en la reproducción de las circunstancias bajo la cuales se desarrolla la situación. El artista añade así un plus de complejidad a los dilemas que el re-enactment de performances plantea a varios niveles (Morgan, 2010; Lydiate y McClean, 2011; Estella, 2015). Y, con ello, se enfrenta a la apertura histórica que supone trabajar en un museo, se inscribe en los tiempos largos que construyen su identidad institucional y testa sus límites como mecanismo patrimonializador.

\section{Todo lo que era sólido...: economías de la presencia}

“¿Cómo puede integrarse en el proceso productivo toda la experiencia del individuo si no es obligado este último a una secuencia de variaciones sobre un tema, performances, improvisaciones? Una secuencia tal, parodiando la autorrealización, marca en realidad la cumbre del sometimiento. Nadie es tan pobre como el que ve su propia relación con la «presencia del otro», es decir su propio cobrar-lengua, reducida a un trabajo asalariado" (Paolo Virno, 2003, pp. 90-91).

La adquisición de las situaciones de Sehgal por parte de importantes museos ha planteado dificultades a sus equipos. La compra de sus piezas supuso cambios en las políticas de algunos centros (Wood, 2013, p. 133; Carpenter, 2014) y, a buen seguro, en determinados contextos - y pienso ahora en España-, la incorporación de obras de Sehgal a una colección pública podría resultar polémica: ¿qué dirían ciertos medios de comunicación —o los contribuyentes por estos atizados- al saberse que su museo ha pagado 100.000 euros por una pieza que se sustancia en... nada? Podría decirse que el artista ha contribuido a abrir un nuevo camino en 
lo que a la comercialización de propuestas no objetuales se refiere y ha modificado los hábitos de compra de algunos grandes museos. Sin embargo, en la actualidad, cuando MoMA, Tate, Walker Art Center, Stedelijk, Van Abbemuseum o FRAC Lorraine, entre otros, han comprado sus situaciones, cabe pensar que el tipo de transacción que estas requieren ha sido institucionalmente sancionado.

Nadie, eso sí, puede hacer suyas las obras de Sehgal. A diferencia de una pieza fluxus o zaj, que cualquiera puede desarrollar a partir de una partitura —ese era el objetivo de Yoko Ono, Walter Marchetti o La Monte Young-, las piezas de Sehgal perderían su sentido fuera de las instituciones museísticas y eventos artísticos con los que él —y sólo él— ha podido negociar las condiciones de producción y recepción de la situación (3). Solo en el museo sus trabajos generan esa incómoda sensación de extrañamiento, muchas de sus situaciones solo significan en el contexto de una colección. Sehgal asume algunas de las máximas duchampianas que se declinaron en varias direcciones en el ámbito de la neovanguardia conceptual: su arte está repleto de dobles sentidos, es antiretiniano, divertido, capaz de desafiar los límites y estructuras del sistema, etc. Pero, al mismo tiempo, su práctica resulta bastante menos democrática y horizontal de lo que pudiera parecer. Está, en ese sentido, lejos de pasar como la vida.

Desde los sesenta y hasta la actualidad, artistas de todo el mundo han adoptado la performance como base para una economía diferente del hecho artístico. Estos performers, comprometidos con el potencial contrainstitucional de un modo de hacer supuestamente accesible y desmaterializado, se recluyeron en el accionismo como una última trinchera desde la cual resistirse a las complicidades entre cultura y capitalismo. Ese espíritu de resistencia se agudizó más si cabe en los años ochenta, coincidiendo con la ofensiva neoliberal, cuando las prácticas conceptuales y performativas perdían su centralidad discursiva al tiempo que sentaban las bases de nuestra contemporaneidad artística. En el contexto español, sin ir más lejos, durante los años ochenta y noventa creció una comunidad de performers -Fernando Baena, Joan Casellas, Nieves Correa, Nélo Vilar, Borja Zabala, entre una extensa y heterogénea nómina de artistas - militantemente duchampiana, que trató de cimentar un arte paralelo a unos circuitos mercantiles que despreciaban y a una institución que les ignoraba. En el marco de un experimentalismo casi extemporáneo, estos concibieron la performance como una práctica no objetual, accesible, abierta, democrática, participativa, poco ambiciosa, repleta de gestos mínimos que contrapesaban la pesadez y grandilocuencia de los discursos en boga. Cualquiera podía hacer performances en unos espacios alternativos carentes de visibilidad, en los que la "calidad artística" era un valor a extinguir. Su arte, este sí, pasaba como la vida misma con el único objetivo de transformarla a través de la acción consciente del sujeto que se atreviese a devenir artista. Esta generación de performers - llamémosla Baena-Zabala - comparte con Sehgal la voluntad de producir un trabajo $100 \%$ inmaterial, aunque sus objetivos son muy distintos. 
Los Baena-Zabala $-\mathrm{y}$, como ellos, muchos otros performers que trabajan en el ámbito de la autogestión en otras latitudes - desarrolló una práctica no objetual y copyleft desde posiciones anticapitalistas: su trabajo podía pagarse, pero su obra no podía venderse. Sehgal, por su parte, ha declarado en varias ocasiones que no tiene ningún problema con la economía de mercado: "critico el modo de producción inherente al objeto material pero no el hecho de que este pueda ser comprado o vendido"(4) (Griffin, 2005, p. 218). El artista constata que las acciones o situaciones existen como tales y que estas pueden circular como mercancías — productos de su autoría - sin necesidad de arrojar rastros objetuales. Altera, con ello, las bases materiales de la economía de mercado (Heiser, 2004, p. 102), y lo hace tratando de eludir sus engranajes tecnológicos - competitividad basada en la reducción de costes de producción, obsolescencia que impulsa el consumo, tecnofilia, etc. - , pero sin pretender una crítica frontal al sistema capitalista ni a los dogmas neoliberales de progreso, crecimiento y libre mercado (Cattelan, 2005, p. 90).

Desde una conciencia ecológica, Sehgal — que tuvo formación en el campo de la economía - experimenta con modos de circulación menos lesivos para el medio ambiente y, en consecuencia, más "interesantes" para nuestras sociedades, lo cual no deja de ser problemático si tenemos en cuenta que el modelo de acumulación basado en la producción y comercialización de conocimiento - eso que vende Sehgal- es, precisamente, el que sostiene el actual capitalismo, tecnológico y financiarizado. Como es sabido, el desarrollo del arte conceptual a finales de los sesenta coincidió con la emergencia de un nuevo modo de acumulación - postindustrial, flexible - en las sociedades capitalistas (Alberro, 2003). Los artistas conceptuales, de manera más o menos consciente, adaptaron su práctica a una nueva realidad mundial moldeada por la revolución de las tecnologías de la información y la comunicación. No parece descabellado pensar que la propuesta de Sehgal responda a un estadio avanzado — quién sabe si final — de ese proceso. Crear algo de la nada, generar significado y valor económico sin necesidad de producir objetos (Hantelmann, 2010, p. 151) es la quintaesencia del capitalismo cognitivo. Hoy, las mercancías no son solo objetos; son, antes que nada, servicios, experiencias, información y conocimiento. Los centros económicos hegemónicos no son aquellos en los que se ensamblan materialmente las mercancías, sino aquellos en que estas se diseñan. Y podría resultar ingenuo pensar que, en esa realidad financiarizada, la producción de conocimiento —en Silicon Valley, por ejemplo - no es contaminante, está desligada de la fabricación de mercancías o representa una vía de salvación ante la catástrofe ecológica que se avecina.

Hay otro aspecto del trabajo de Sehgal que guarda una relación ambivalente con el capitalismo actual: la producción de subjetividad. Podríamos pensar que Sehgal consigue proveer a los sujetos que aceptan interaccionar con sus intérpretes de experiencias que les ayudan a salir de su pasividad — como 
visitantes de un museo- para convertirse en ciudadanos más activos, conscientes e, incluso, responsables (Hantelmann, 2010, pp. 171-173); y, en cierto modo, así es. No podemos obviar, no obstante, que esas situaciones son, por lo general, experiencias individuales, estandarizadas y administradas en el marco de una estructura institucional. Si valoramos de manera dialéctica los trabajos de Sehgal, es fácil darse cuenta de que algunos de los elementos emancipadores que el artista parece potenciar, tales como la creatividad, la capacidad de negociar situaciones imprevisibles, la aparente precariedad material y el carácter casual y desenfadado de sus propuestas, son elementos claves en los modos de subjetivación del capitalismo postindustrial, empeñado en convertir cada momento de nuestras vidas en tiempo productivo. Para ello el sistema requiere individuos activos, creativos, ávidos de consumo, capaces de responder ante situaciones cambiantes $\mathrm{y}$, a ser posible, felices pese al estado de precariedad vital al que la flexibilidad les condena. Así, la actividad del artista parece adecuarse al tipo de trabajador que requiere hoy el capitalismo - un emprendedor nómada, incansable publicista de sí-, al tiempo que su práctica contribuye a socializar a los sujetos, su público, sus visitantes, como productores-consumidores (Kunst, 2015). Desde esta perspectiva, el arte de Sehgal, como buena parte de las prácticas contemporáneas, también pasa como la vida, en el peor de los sentidos: arte $=$ vida $=$ trabajo $=$ arte .

Por supuesto, Sehgal solo trata de pensar una posible vía de experimentación en el ámbito del museo, que concibe como "un lugar para políticas a largo plazo"(5) (Griffin, 2005, p. 219). Su propuesta se mueve entre dos dimensiones: la dimensión simbólica sobre la que se asienta todo trabajo artístico - así podrían hacerse las cosas, desde aquí podríamos pensarlas de otra manera-, y la estéticofactual - así pasan las cosas - que se concreta en el encuentro entre intérpretes y visitantes en las salas de un museo. Ahora bien, su intento por desterrar el objeto de su praxis a través de una política de la inmediatez no solo requiere múltiples procesos de mediación, sino que, además, también pone sobre la mesa toda una serie de cuestiones relacionadas con problemas materiales: ¿cuánto cobran sus intérpretes por convertirse en "Sehgals" (C), no pictures); por compartir su tiempo, presencia, afectividad, inteligencia, desnudez; por perder su voz para devenir la obra de otro?, ¿acaso no están estos ensamblando un proyecto que genera beneficios - más o menos elevados - al autor de un conocimiento copyright?, ¿cuántos viajes - poco importa con qué medio - ha tenido que realizar Sehgal o su equipo para negociar y supervisar su año en el Stedelijk?, ¿cuánta energía — calorías, kilovatios - se ha consumido y cuánta polución —emisiones de $\mathrm{CO} 2$ - se ha producido en esos doce meses? Tal vez estas preguntas resulten capciosas, pero pueden ayudarnos a percibir la materialidad de una propuesta que se levanta sobre una inmaterialidad pretendidamente liberadora.

Parte del éxito cosechado por Sehgal en el sistema internacional del arte reside en el renovado interés de las instituciones por las poéticas experienciales, 
que alimentan un acelerado proceso de "eventificación" en sus programaciones. El tipo de delegación que Sehgal propone es el paradigma de performance que circula en exposiciones blockbuster como 11-15 Rooms. En este proyecto, dos afamados comisarios, Klaus Biesenbach y Hans Ulrich Obrist, seleccionaron a una nómina creciente y cambiante de artistas que presentaron performances de naturaleza delegada en habitaciones independientes (Biesenbach y Obrist, 2013, 2014). 11-15 Rooms es un producto itinerante, mediático, estandarizado, modular y flexible, capaz de adaptarse a contextos muy diferentes (6). Las trayectorias y red de contactos de sus comisarios así como el prestigio de los artistas aseguraban una buena difusión, recepción crítica y cifras de visitantes. En la rueda de prensa de 14 Rooms, acogida en Basilea por la Fundación Beyeler, Theater Basel y Art Basel, con un diseño arquitectónico de Herzog \& de Meuron, el director de la feria, Marc Spiegler, explicó con claridad por qué esta apoyaba unas prácticas que, en origen, se habrían posicionado contra el mercado. La feria estaría interesada en el arte más innovador, la performance habría ganado peso en los discursos artísticos y muchos creadores trabajan hoy en su puesta el día desde nuevos códigos experienciales. Del mismo modo que Basel apostó por la fotografía y contribuyó a su expansión comercial veinticinco años atrás, ahora da visibilidad a prácticas performativas en las que se adivina un importante nicho de negocio. En ese territorio, nuestro protagonista ha conseguido generar una potente imagen de marca basada en un modo de hacer compartido por otros artistas. De alguna manera, su presencia internacional y el aura que envuelve su trabajo tienden a fagocitar cualquier propuesta coreográfica hasta dar lugar a lo que Bishop denomina "Sehgal effect": "desgraciadamente, todo trabajo de danza que se desarrolle en un espacio artístico hoy se parece a y se percibe como un Tino Sehgal, aunque el fondo sea muy distinto" (7) (2014a, p. 66).

El artista, sin embargo, parece no haber inventado nada nuevo. Resulta tan fácil como tramposo rastrear los posibles antecedentes de una práctica - ya sea la delegación de performances o la venta de estas a través de acuerdos orales-, así que no voy a ahorrar al lector el esfuerzo de buscar genealogías en las que insertar estas propuestas. Está claro, en cualquier caso, que Sehgal nos ofrece un producto atractivo, que no es nuevo, pero que lo parece al adaptar formas de trabajo artístico que operaban en el sistema desde hacía varias décadas a las dinámicas económicas y a los intereses de las instituciones artísticas. En ese sentido, la laxa e ineficaz prohibición de hacer fotos a sus piezas ha sido interpretada como una estrategia de promoción (Bishop, 2004, p. 216) o, incluso, como "otra restricción arbitraria para intensificar el deseo de sus visitantes hacia su trabajo: unas esposas de terciopelo, un cinturón de castidad" (8) (Davis, 2010). La importancia de la inmediatez en la recepción y valoración de su obra y el deseo de desplazar a la imagen como principal fuente de información otorgan al relato de la experiencia una nueva centralidad. El hecho de que, como decía Vila-Matas, 
"solo participando en su performance uno pudiera decir que había visto la obra" genera una atracción por ver, conocer y discutir - consumir - un producto en varios sentidos inmaterial. Quizás aquí resida una de las principales aportaciones de Sehgal. Su obra se adhiere al relato para cuestionarlo, reta nuestra capacidad de verbalizar una situación artística, nos incita a pensar - sin la referencia material de un objeto, una imagen, un catálogo o un documento - en aquello que nace del encuentro entre nuestros cuerpos y los cuerpos de sus intérpretes. No en vano su trabajo ha generado abundante literatura crítica, la mayor parte de la cual se basa en la narración — siempre insatisfactoria - de una experiencia; algo a lo que este texto, me temo, no ha podido escapar.

\section{Notas}

(1) "I would like to do a solo exhibition in a totally empty museum which at the same time would be full, empty and full since there would be no objects whatsoever, but there would be loads of pieces going on and in each room, so in some sense it would be very conventional".

(2) "we could develop it along the way with Tino and we could every month choose new works by him and with him, and new combinations of works, which could also build on the experience which we had with works during the previous month. So we could develop it quite naturally. It was work in progress, a real dialogue with the artist. For this plan, we found the budget in three months; because of the daring set up, it was generously supported by a couple of funds. It was quite a challenge to organise it".

(3) Podríamos considerar la inclusión de la pieza de Sehgal you are already doing all of it (2002) en el proyecto do it de Hans Ulrich Obrist como la excepción que confirma la regla (Obrist, 2013, p. 350). La página dedicada a Seghal en este compendio de instrucciones reproduce el título de la obra: "you are already doing all of it".

(4) "I criticize the mode of production inherent to a material object but not the fact that it can be bought or sold".

(5) "as a place for long-term politics".

(6) La serie de exposiciones cuenta, por el momento, con cinco entregas: 11 Rooms, Manchester International Festival, Manchester, 2011; 12 Rooms, Ruhrtriennale, Essen, 2012; 13 Rooms, Public Art Projects, Sidney, 2013; 14 Rooms, Art Basel, Basilea, 2014; 15 Rooms, Long Museum, Shanghai, 2015. Entre los artistas destacan Marina Abramovic, Allora/Calzadilla, John Baldessari, Dominique Gonzalez-Foerster, Damien Hirst, Joan Jonas, Roman Ondak, Tino Sehgal o Santiago Sierra.

(7) "unfortunately, every instance of dancing in the gallery now looks and feels like a Tino Sehgal, even the content is wildly different".

(8) "another arbitrary restraint to intensify his visitors' desire for his work: a pair of velvet handcuffs; a chastity belt". 


\section{Referencias bibliográficas}

Albarrán Diego, J. (2012). La fotografía ante el arte de acción. Efímera, 3.

Alberro, A. (2003). Conceptual Art and the Politics of Publicity. Cambridge: MIT. Auslander, P. (1999). Liveness: Performance in a Mediatized Culture. New York: Routledge.

Auslander, P. (2006). The Performativity of Performance Documentation. PAJ, 28(3).

Auslander, P. (2014). Surrogate Performances. Performance, Documentation and the New York Avant-Garde ca. 1964-1974. En E. Carpenter (ed), On Performativity. Minneapolis: Walker Art Center. Recuperado de http:// www.walkerart.org/collections/publications/performativity/surrogateperformances/

Biesenbach, K.; Obrist H.U. (2013). 13 Rooms. Sydney, Kaldor Public Art Projects.

Biesenbach, K.; Obrist H.U. (2014). 14 Rooms. Basel: Hatje Cantz.

Bishop, C. (2005). No Pictures, Please. Artforum, mayo.

Bishop. C. (2014a). The Perils and Possibilities of Dance in the Museum: Tate, MoMA, and Whitney. Dance Research Journal, 46(3).

Bishop. C. (2014b). Je ne veux pas de rétrospective: Xavier Le Roy et l'exposition en tant que médium. En B. Cvejic (ed), Rétrospective par Xavier Le Roy. Paris: Les Presses du Réel.

Cahier (2015). Cahier Interviews Beatrix Ruf. Cahier, 27. Recuperado de http:// www.cahier-online.de/news/cahier27-news_e.html

Carpenter, E. (2014). Be the Work: Intersubjectivity in Tino Sehgal's This objective of that object. En E. Carpenter (ed), On Performativity. Minneapolis: Walker Art Center. Recuperado de http://walkerart.org/collections/publications/ performativity/be-the-work.

Cattelan, M. (2009). Tino Sehgal: Economics of Progress. Flash Art, 264.

Christov-Bakargiev, C. (2012). dOCUMENTA (13). The Guidebook. Catalog 3/3. Kassel: dOCUMENTA, Hatje Cantz.

Davis, B. (2010). Photos for Tino. Artnet. Recuperado de http://www.artnet.com/ magazineus/reviews/davis/tino-sehgal1-7-10.asp

Estella, I. (2015). El arte de performance a debate: originalidad, re-escenificación y los modelos alternativos de George Maciunas. Ars Longa, 24.

Griffin, T. (2005). Tino Sehgal: An Interview. Artforum, mayo.

Heiser, J. (2004). Tino Sehgal. En Funky Lessons. Berlín: BüroFriedrich.

Hinojosa, L. (2015). Objetualizar la experiencia, historiar la ausencia. Notas sobre performance y museos. En J. Albarrán e I. Estella (eds), Llámalo performance: historia, disciplina y recepción. Madrid: Brumaria. 
Kravchuk, D. (2016). Tino Sehgal. A Year in the Stedelijk. Mu[see]um. Recuperado de http://www.museeum.net/article/188/martijn-van-niewenhuyzen.html

Kunst, B. (2015). Artist at Work. Proximity of Art and Capitalism. Winchester, Washington: Zero Books.

Lydiate, H.; McClean, D. (2011). Re-Performance Art. Art Monthly, 349.

Morgan, R.C. (2010). Thoughts on Re-performance, Experience, and Archivism. $P A J, 32(3)$.

Obrist, H.U. (2003). Hans Ulrich Obrist Interviews Tino Sehgal. En Katalog des Kunstpreises der Böttcherstaße. Bremen: Kunsthalle.

Obrist, H.U. (2013). do it. The Compendium. New York: Independent Curators International.

Richard, S. (2009). Unconcealed. The International Network of Conceptual Artists 1967-1977. Dealers, Exhibitions and Public Collections. London: Ridinghouse, Norwich University College of the Arts.

Vila-Matas, E. (2014). Kassel no invita a la lógica. Barcelona: Seix Barral.

Virno, P. (2003). Virtuosismo y revolución: notas sobre el concepto de acción política. En Virtuosismo y revolución. La acción política en la era del desencanto. Madrid: Traficantes de Sueños.

Von Hantelmann, D. (2010). How to Do Things with Art. Zurich: JRP / Ringier.

Wood, C. (2014). In Advance of the Broken Arm: Collecting Live Art and the Museum's Changing Game. En T. Calonje (ed), Live Forever. Collecting Live Art. London: Koenig.

\section{Cita Recomendada}

Albarrán Diego, J. (2017). Sehgal no invita a la lógica. Performance, experiencia y economía inmaterial en Tino Sehgal. Sin Objeto, 00, 24-39. Doi: http://dx.doi.org/10.18239/sinobj_2017.00.02 


\section{Biografía}

\section{Juan Albarrán Diego}

Universidad Autónoma de Madrid

Juan.albarran@uam.es

Juan Albarrán es doctor en Historia del Arte por la Universidad de Salamanca y profesor ayudante doctor en el Departamento de Historia y Teoría del Arte de la Universidad Autónoma de Madrid. Ha impartido docencia en las universidades de Castilla-La Mancha y Salamanca y, desde 2011, colabora en el programa de Duke University, Madrid. Forma parte del equipo docente del Máster en Historia del Arte Contemporáneo y Cultura Visual (UAM / UCM / Museo Reina Sofía), del que es coordinador. Sus líneas de investigación se centran en las prácticas y discursos artísticos de la contemporaneidad, especialmente en el contexto postransicional español y en los ámbitos de la fotografía y la performance. Sobre estas cuestiones, ha editado los libros Arte y Transición (2012) y Llámalo Performance: historia, disciplina y recepción (con Iñaki Estella, 2015). 